\title{
Bessel 函数的一个积分估计及其应用
}

丁勇*, 李再

北京师范大学数学科学学院, 数学与复杂系统教育部重点实验室, 北京 100875

*E-mail: dingy@bnu.edu.cn

收稿日期: 2006-01-07; 接受日期: 2007-09-16

国家自然科学基金 (批准号: 10571015) 和教育部博士点基金 (批准号: 20050027025) 资助项目

摘要推广了 Calderón-Zygmund 的结果, 给出一个新的 Bessel 函数积分估计. 应用这个 结果证明了变量核的参数型 Marcinkiewicz 积分 $\mu_{\Omega}^{\rho}$ 的 $L^{2}$ 有界性, 其中核函数 $\Omega$ 在 $\mathbb{R}^{n}$ 的 单位球面 $S^{n-1}$ 上没有任何光滑性.

关键词 Bessel 函数 Marcinkiewicz 积分变量核

$\operatorname{MSC}(2000)$ 主题分类 $42 \mathrm{~B} 30,42 \mathrm{~B} 99$

\section{1 引言}

当 $R(\nu)>-\frac{1}{2}$ 时, Bessel 函数 $J_{\nu}(t)$ 定义为

$$
J_{\nu}(t)=\frac{(t / 2)^{\nu}}{\Gamma(\nu+1 / 2) \Gamma(1 / 2)} \int_{-1}^{1}\left(1-s^{2}\right)^{\nu-1 / 2} \mathrm{e}^{i t s} d s .
$$

众所周知, Bessel 函数 $J_{\nu}(t)$ 在分析学中具有重要应用. 1955 年, Calderón 和 Zygmund 给出了下面 Bessel 函数的一个积分估计:

定理 $\mathbf{A}^{[1]}$ 对于 $\lambda=(n-2) / 2$, 存在 $C>0$, 使得对任意 $h \geqslant 0$ 及 $m=1,2, \ldots$, 有

$$
\left|\int_{0}^{h} \frac{J_{m+\lambda}(t)}{t^{\lambda+1}} d t\right| \leqslant \frac{C}{m^{\lambda+1}}
$$

Calderón 和 Zygmund ${ }^{[1]}$ 运用这个估计给出了一类带变量核的奇异积分算子的 $L^{2}$ 有界 性, 它密切联系着变系数的二阶线性椭圆方程解的正则性问题.

我们首先给出定理 $\mathrm{A}$ 的推广.

定理 1 对于 $\lambda=(n-2) / 2$ 且 $-\lambda \leqslant \alpha \leqslant 1$, 存在 $C>0$, 使得对任意的 $h \geqslant 0$ 及 $m=1,2, \ldots$, 有

$$
\left|\int_{0}^{h} \frac{J_{m+\lambda}(t)}{t^{\lambda+\alpha}} d t\right| \leqslant \frac{C}{m^{\lambda+\alpha}}
$$


运用上面的定理我们将证明带变量核的参数型 Marcinkiewicz 积分 $\mu_{\Omega}^{\rho}(0<\rho \leqslant n / 2)$ 在 $L^{2}\left(\mathbb{R}^{n}\right)$ 上的有界性. 在阐述这个结论前, 首先给出变量核的参数型 Marcinkiewicz 积分 $\mu_{\Omega}^{\rho}$ 的 定义.

令 $S^{n-1}$ 为 $\mathbb{R}^{n}(n \geqslant 2)$ 上的单位球面. 若 $\Omega(x, z)$ 满足下面的条件:

(i) 对任意 $x, z \in \mathbb{R}^{n}$ 及 $\lambda>0$, 有 $\Omega(x, \lambda z)=\Omega(x, z)$;

(ii) $\|\Omega\|_{L^{\infty}\left(\mathbb{R}^{n}\right) \times L^{q}\left(S^{n-1}\right)}:=\sup _{x \in \mathbb{R}^{n}}\left(\int_{S^{n-1}}\left|\Omega\left(x, z^{\prime}\right)\right|^{q} d \sigma\left(z^{\prime}\right)\right)^{1 / q}<\infty$, 其中 $z^{\prime}=z /|z|, z \in$ $\mathbb{R}^{n} \backslash\{0\}$,

则称 $\mathbb{R}^{n} \times \mathbb{R}^{n}$ 上的函数 $\Omega(x, z) \in L^{\infty}\left(\mathbb{R}^{n}\right) \times L^{q}\left(S^{n-1}\right)(q \geqslant 1)$.

变量核的参数型 Marcinkiewicz 积分 $\mu_{\Omega}^{\rho}$ 的定义为

$$
\mu_{\Omega}^{\rho}(f)(x)=\left(\int_{0}^{\infty}\left|F_{\Omega, t}^{\rho}(x)\right|^{2} \frac{d t}{t}\right)^{1 / 2},
$$

其中

$$
F_{\Omega, t}^{\rho}(x)=\frac{1}{t^{\rho}} \int_{|x-y| \leqslant t} \frac{\Omega(x, x-y)}{|x-y|^{n-\rho}} f(y) d y \quad(0<\rho<n)
$$

且

$$
\int_{S^{n-1}} \Omega\left(x, z^{\prime}\right) d \sigma\left(z^{\prime}\right)=0, \quad x \in \mathbb{R}^{n} .
$$

当 $\rho=1$ 时, 我们把 $\mu_{\Omega}^{1}$ 简记为 $\mu_{\Omega}$.

历史上, Stein ${ }^{[2]}$ 首先定义并研究了高维卷积核 Marcinkiewicz 积分算子 $\mu_{\Omega}$, 即 $\Omega(x, z)=$ $\Omega(z)$ 的情形. 有关卷积核的 $\mu_{\Omega}$ 的有界性可见文献 [3-6]. 最近, 在文献 [7] 中作者得到了下面 的变量核 Marcinkiewicz 积分 $\mu_{\Omega}$ 的 $L^{2}$ 有界性结果:

定理 $\mathbf{B}^{[7]}$ 若 $\Omega \in L^{\infty}\left(\mathbb{R}^{n}\right) \times L^{q}\left(S^{n-1}\right)$ 且满足 (1.3) 式, 其中 $q>2(n-1) / n$, 则 $\mu_{\Omega}$ 是 $(2,2)$ 型的.

另一方面, 1960 年 Hörmander ${ }^{[8]}$ 引进并给出了卷积核的参数型 Marcinkiewicz 积分的 $L^{p}$ 有界性. 因此, 一个自然的问题就是定理 B 对于变量核的参数型 Marcinkiewicz 积分 $\mu_{\Omega}^{\rho}$ 是否 仍成立. 本文的第二个结果将对其给出肯定的回答.

定理 2 若 $0<\rho \leqslant n / 2$, 则在与定理 $\mathrm{B}$ 相同的条件下, 变量核的参数型 Marcinkiewicz 积分 $\mu_{\Omega}^{\rho}$ 是 $(2,2)$ 型的.

\section{2 定理 1 的证明}

先回顾 Bessel 函数 $J_{\nu}$ 的一些性质, 其证明可见文献 [9].

(a) $t J_{\nu}^{\prime}(t)+\nu J_{\nu}(t)=t J_{\nu-1}(t)$ ，其中 $R(\nu)>\frac{1}{2}$;

(b) $t J_{\nu}^{\prime}(t)-\nu J_{\nu}(t)=-t J_{\nu+1}(t)$;

(c) $\int_{0}^{1} J_{\nu}(\nu t) d t \sim \frac{1}{3 \nu}-\frac{2^{2 / 3}}{3^{1 / 3} \cdot 5 \Gamma(1 / 3) \nu^{5 / 3}} ;$

(d) $\int_{0}^{\infty} \frac{J_{\nu}(t) d t}{t^{\nu-\mu+1}} \leqslant \frac{C \cdot \Gamma\left(\frac{1}{2} \mu\right)}{2^{\nu-\mu+1} \Gamma\left(\nu-\frac{1}{2} \mu+1\right)}$, 其中 $R(\mu)<R\left(\nu+\frac{3}{2}\right)$;

(e) $J_{\mu+\nu+1}(t)=\frac{t^{\mu+1}}{2^{\mu} \Gamma(\mu+1)} \int_{0}^{\pi / 2} J_{\nu}(t \sin \theta) \sin ^{\nu+1} \theta \cos ^{2 \mu+1} \theta d \theta$.

定理 1 的证明还需要下面两个引理:

引理 2.1 对任意 $-\lambda \leqslant \alpha \leqslant 1, \lambda=(n-2) / 2$ 及 $n \geqslant 2$,

(i) $\int_{0}^{\nu} J_{\nu}(t) d t \leqslant \frac{1}{3}$; 
(ii) $\frac{J_{\nu}(t)}{t^{\lambda+\alpha}}=-\frac{J_{\nu}^{\prime}(t)}{t^{\lambda+\alpha-1}\left(t^{2}-\nu^{2}\right)}-\frac{J_{\nu}^{\prime \prime}(t)}{t^{\lambda+\alpha-2}\left(t^{2}-\nu^{2}\right)}$, 其中 $R(\nu)>\frac{1}{2}$.

证明 应用性质 (c) 即可得到 (i). 下面给出 (ii) 的证明. 由性质 (a) 有

$$
J_{\nu}^{\prime}(t)+t J_{\nu}^{\prime \prime}(t)+\nu J_{\nu}^{\prime}(t)=J_{\nu-1}(t)+t J_{\nu-1}^{\prime}(t) .
$$

由 (a) 和 (b) 易知,

$$
\begin{aligned}
& J_{\nu-1}(t)=J_{\nu}^{\prime}(t)+\frac{\nu}{t} J_{\nu}(t) \\
& t J_{\nu-1}^{\prime}(t)=(\nu-1) J_{\nu-1}(t)-t J_{\nu}(t) .
\end{aligned}
$$

从 (2.1)-(2.3) 式可以得到

$$
\begin{aligned}
J_{\nu}^{\prime}(t)+t J_{\nu}^{\prime \prime}(t)+\nu J_{\nu}^{\prime}(t) & =J_{\nu}^{\prime}(t)+\frac{\nu}{t} J_{\nu}(t)+(\nu-1) J_{\nu-1}(t)-t J_{\nu}(t) \\
& =J_{\nu}^{\prime}(t)+\frac{\nu}{t} J_{\nu}(t)+(\nu-1)\left(J_{\nu}^{\prime}(t)+\frac{\nu}{t} J_{\nu}(t)\right)-t J_{\nu}(t) .
\end{aligned}
$$

从而,

$$
t J_{\nu}^{\prime \prime}(t)=-J_{\nu}^{\prime}(t)+\frac{\nu^{2}}{t} J_{\nu}(t)-t J_{\nu}(t) .
$$

这样由 (2.4) 式即可得 (ii).

引理 2.2 对于 $\lambda=(n-2) / 2, n \geqslant 2$, 存在常数 $C>0$, 使得对任意 $-\lambda \leqslant \alpha \leqslant 1$ 及 $m=1,2, \ldots$,

$$
\left|\int_{0}^{\infty} \frac{J_{m+\lambda}(t)}{t^{\lambda+\alpha}} d t\right| \leqslant \frac{C}{m^{\lambda+\alpha}}
$$

证明 由性质 (d) 有

$$
\left|\int_{0}^{\infty} \frac{J_{m+\lambda}(t)}{t^{\lambda+\alpha}} d t\right| \leqslant \frac{C \cdot \Gamma((m+1-\alpha) / 2)}{2^{\lambda+\alpha} \Gamma(m / 2+\lambda+\alpha / 2+1 / 2)} .
$$

应用 Stirling 公式, 当 $x>1$ 时我们得到

$$
\sqrt{2 \pi} x^{x-1 / 2} \mathrm{e}^{-x} \leqslant \Gamma(x) \leqslant 2 \sqrt{2 \pi} x^{x-1 / 2} \mathrm{e}^{-x} .
$$

利用 (2.5) 式并注意到 $m+2 \lambda+\alpha+1 \geqslant m+1-\alpha$, 可得

$$
\begin{aligned}
& \left|\int_{0}^{\infty} \frac{J_{m+\lambda}(t)}{t^{\lambda+\alpha}} d t\right| \\
& \quad \leqslant \frac{C \cdot \sqrt{2 \pi}[(m+1-\alpha) / 2)]^{m / 2+1 / 2-\alpha / 2-1 / 2} \mathrm{e}^{-(m+1-\alpha) / 2}}{2^{\lambda+\alpha} \sqrt{2 \pi}(m / 2+\lambda+\alpha / 2+1 / 2)^{m / 2+\lambda+\alpha / 2+1 / 2-1 / 2} \mathrm{e}^{-(m / 2+\lambda+\alpha / 2+1 / 2)}} \\
& \quad=C \cdot \mathrm{e}^{\lambda+\alpha} \frac{(m+1-\alpha)^{m / 2-\alpha / 2}}{(m+2 \lambda+\alpha+1)^{m / 2+\lambda+\alpha / 2}} \\
& \quad=C \cdot \mathrm{e}^{\lambda+\alpha}(m+1-\alpha)^{-\lambda-\alpha} \frac{(m+1-\alpha)^{m / 2+\lambda+\alpha / 2}}{(m+2 \lambda+\alpha+1)^{m / 2+\lambda+\alpha / 2}} \\
& \quad \leqslant \frac{C}{m^{\lambda+\alpha}} .
\end{aligned}
$$

引理得证.

定理 1 的证明 证明的主要思想源自文献 [1]. 令 $\nu=m+\lambda$. 考虑如下 4 种情形, 即:

$$
1^{\circ} 0 \leqslant h \leqslant \nu / 2 ; \quad 2^{\circ} \nu / 2 \leqslant h \leqslant \nu ; \quad 3^{\circ} \nu \leqslant h \leqslant 2 \nu ; \quad 4^{\circ} h \geqslant 2 \nu .
$$

情形 $1^{\circ}: 0 \leqslant h \leqslant \nu / 2$. 由 $J_{\nu}$ 的定义有

$$
\left|J_{\nu}(t)\right| \leqslant\left|\frac{(t / 2)^{\nu}}{\Gamma(\nu+1 / 2) \Gamma(1 / 2)} \int_{-1}^{+1}\left(1-s^{2}\right)^{\nu-1 / 2} \mathrm{e}^{i s t} d s\right|
$$




$$
\begin{aligned}
& \leqslant \frac{(t / 2)^{\nu}}{\Gamma(\nu+1 / 2) \sqrt{\pi}} \int_{-1}^{+1}\left(1-s^{2}\right)^{\nu-1 / 2} d s \\
& \leqslant \frac{C(t / 2)^{\nu}}{\Gamma(\nu+1 / 2)} .
\end{aligned}
$$

因此

$$
\left|\int_{0}^{h} \frac{J_{m+\lambda}(t)}{t^{\lambda+\alpha}} d t\right| \leqslant \frac{C(1 / 2)^{\nu}}{\Gamma(\nu+1 / 2)} \int_{0}^{h} \frac{t^{\nu}}{t^{\lambda+\alpha}} d t \leqslant \frac{C}{2^{\nu} \Gamma(\nu+1 / 2)} \int_{0}^{h} t^{m-\alpha} d t .
$$

容易看出 $\left(\frac{\mathrm{e}}{4}\right)^{m+\lambda} \cdot(m+\lambda)$ 有界. 由 $(2.5)$ 式可得

$$
\begin{aligned}
\frac{C}{2^{\nu} \Gamma(\nu+1 / 2)} \int_{0}^{h} t^{m-\alpha} d t & \leqslant \frac{C}{2^{m+\lambda} \Gamma(\nu+1 / 2)} \cdot \frac{1}{m-\alpha+1}\left(\frac{m+\lambda}{2}\right)^{m-\alpha+1} \\
& \leqslant \frac{C}{2^{m+\lambda} \sqrt{2 \pi}(\nu+1 / 2)^{\nu+1 / 2-1 / 2} \mathrm{e}^{-\nu-1 / 2}} \cdot \frac{1}{m-\alpha+1}\left(\frac{m+\lambda}{2}\right)^{m-\alpha+1} \\
& \leqslant \frac{C}{2^{2 m+\lambda-\alpha+1}} \cdot \frac{(m+\lambda)^{m-\alpha+1}}{(m+\lambda+1 / 2)^{m+\lambda}} \cdot \frac{\mathrm{e}^{m+\lambda+1 / 2}}{m-\alpha+1} \\
& \leqslant \frac{C \cdot \mathrm{e}^{m+\lambda+1 / 2}}{4^{m+\lambda / 2-\alpha / 2+1 / 2}} \cdot(m+\lambda) \cdot(m+\lambda)^{-\lambda-\alpha} \frac{1}{m-\alpha+1} \\
& \leqslant C\left(\frac{\mathrm{e}}{4}\right)^{m+\lambda} \cdot(m+\lambda) \cdot \frac{1}{4^{-\lambda / 2-\alpha / 2+1 / 2}} \cdot \frac{(m+\lambda)^{-\lambda-\alpha}}{m-\alpha+1} \\
& \leqslant \frac{C}{(m+\lambda)^{\lambda+\alpha}} .
\end{aligned}
$$

情形 $2^{\circ}: \quad \nu / 2 \leqslant h \leqslant \nu$. 注意到当 $0<t \leqslant \nu$ 时 $J_{\nu}(t)>0$ (见文献 [9, p. 254]), 由引理 $2.1(\mathrm{i}$ ) 有

$$
\int_{\nu / 2}^{h} \frac{J_{\nu}(t)}{t^{\lambda+\alpha}} d t \leqslant C \nu^{-\lambda-\alpha} \int_{\nu / 2}^{h} J_{\nu}(t) d t \leqslant C \nu^{-\lambda-\alpha} \int_{0}^{\nu} J_{\nu}(t) d t \leqslant C m^{-\lambda-\alpha}
$$

结合情形 $1^{\circ}$ 的结果, 可以得到

$$
\int_{0}^{h} \frac{J_{\nu}(t)}{t^{\lambda+\alpha}} d t=\int_{0}^{\nu / 2} \frac{J_{\nu}(t)}{t^{\lambda+\alpha}} d t+\int_{\nu / 2}^{h} \frac{J_{\nu}(t)}{t^{\lambda+\alpha}} d t \leqslant C m^{-\lambda-\alpha} .
$$

情形 $4^{\circ}: h \geqslant 2 \nu$. 由引理 2.2 , 只需证明

$$
\left|\int_{h}^{\infty} \frac{J_{m+\lambda}(t)}{t^{\lambda+\alpha}} d t\right| \leqslant \frac{C}{m^{\lambda+\alpha}}
$$

因为 $\left|J_{\nu}^{\prime}(t)\right| \leqslant 1$ (见文献 $[1$, p. 221]) 且 $h \geqslant 2 \nu$, 有

$$
\int_{h}^{\infty}\left|\frac{J_{\nu}^{\prime}(t)}{t^{\lambda+\alpha-1}\left(t^{2}-\nu^{2}\right)}\right| d t \leqslant \int_{h}^{\infty} \frac{d t}{t^{\lambda+\alpha-1}\left(t^{2}-\nu^{2}\right)} \leqslant C \int_{h}^{\infty} \frac{d t}{t^{\lambda+\alpha+1}} \leqslant C m^{-\lambda-\alpha} .
$$

另一方面, 对任意 $A>h$, 存在 $\xi$ 满足 $h<\xi<A$, 使得

$$
\begin{aligned}
\left|\int_{h}^{A} \frac{J_{\nu}^{\prime \prime}(t)}{t^{\lambda+\alpha-2}\left(t^{2}-\nu^{2}\right)} d t\right| & \leqslant\left|\frac{1}{h^{\lambda+\alpha-2}\left(h^{2}-\nu^{2}\right)} \int_{h}^{\xi} J_{\nu}^{\prime \prime}(t) d t\right|+\left|\frac{1}{A^{\lambda+\alpha-2}\left(A^{2}-\nu^{2}\right)} \int_{\xi}^{A} J_{\nu}^{\prime \prime}(t) d t\right| \\
& \leqslant C h^{-\lambda-\alpha}\left|\int_{h}^{\xi} J_{\nu}^{\prime \prime}(t) d t\right|+C A^{-\lambda-\alpha}\left|\int_{\xi}^{A} J_{\nu}^{\prime \prime}(t) d t\right| \\
& \leqslant C h^{-\lambda-\alpha}\left|J_{\nu}^{\prime}(\xi)-J_{\nu}^{\prime}(h)\right|+C A^{-\lambda-\alpha}\left|J_{\nu}^{\prime}(A)-J_{\nu}^{\prime}(\xi)\right|
\end{aligned}
$$

82 


$$
\leqslant C\left(h^{-\lambda-\alpha}+A^{-\lambda-\alpha}\right)
$$

其中 $C$ 不依赖于 $\xi$. 由于 $\lambda+\alpha>0$, 令 $A \rightarrow \infty$, 可以得到

$$
\left|\int_{h}^{\infty} \frac{J_{\nu}^{\prime \prime}(t)}{t^{\lambda+\alpha-2}\left(t^{2}-\nu^{2}\right)} d t\right| \leqslant C h^{-\lambda-\alpha} .
$$

从而, 由引理 2.1 (ii), (2.8) 和 (2.9) 式得

$$
\left|\int_{h}^{\infty} \frac{J_{\nu}(t)}{t^{\lambda+\alpha}} d t\right| \leqslant\left|\int_{h}^{\infty} \frac{J_{\nu}^{\prime}(t)}{t^{\lambda+\alpha-1}\left(t^{2}-\nu^{2}\right)}\right|+\left|\int_{h}^{\infty} \frac{J_{\nu}^{\prime \prime}(t)}{t^{\lambda+\alpha-2}\left(t^{2}-\nu^{2}\right)} d t\right| \leqslant C m^{-\lambda-\alpha} .
$$

情形 $3^{\circ}: \nu \leqslant h \leqslant 2 \nu$. 由积分第二中值定理

$$
\int_{\nu}^{h} \frac{J_{\nu}(t)}{t^{\lambda+\alpha}} d t=\nu^{-\lambda-\alpha} \int_{\nu}^{\xi} J_{\nu}(t) d t \quad(\nu<\xi<2 \nu),
$$

因而只需证明等式右边的积分有界. 令 $t^{\prime}=t / \nu$,

$$
\int_{\nu}^{\xi} J_{\nu}(t) d t=\nu \int_{1}^{\xi / \nu} J_{\nu}\left(\nu t^{\prime}\right) d t^{\prime} .
$$

由文献 [1, p. 221] 中的 (6.2) 式, 可以得到

$$
\int_{1}^{\xi / \nu} J_{\nu}\left(\nu t^{\prime}\right) d t^{\prime}=O\left(\frac{1}{\nu}\right) .
$$

从而完成了定理的证明.

\section{3 定理 2 的证明}

引理 3.1 设 $0<\rho \leqslant \frac{n}{2}$ 且 $g_{\rho}(f)(x)=\left(\int_{0}^{\infty}\left|N_{\varepsilon}^{\rho} f(x)\right|^{2} \frac{d \varepsilon}{\varepsilon}\right)^{1 / 2}$, 其中 $N_{\varepsilon}^{\rho}$ 满足

$$
\left(N_{\varepsilon}^{\rho} f\right)^{\wedge}(x)=\frac{1}{(\varepsilon|x|)^{\rho}} \int_{0}^{\varepsilon|x|} \frac{J_{m+\lambda}(t)}{t^{\lambda-\rho+1}} d t \cdot \hat{f}(x),
$$

这里 $J_{\nu}$ 为 Bessel 函数, $m \in \mathbb{N}$, 则存在与 $m$ 无关的常数 $C>0$, 使得

$$
\left\|g_{\rho}(f)\right\|_{L^{2}} \leqslant \frac{C}{m^{\lambda+1}}\|f\|_{L^{2}}
$$

证明 令

$$
\eta(|x|)=\frac{1}{|x|^{\rho}} \int_{0}^{|x|} \frac{J_{m+\lambda}(t)}{t^{\lambda-\rho+1}} d t .
$$

运用 Plancherel 定理得到

$$
\begin{aligned}
\left\|g_{\rho}(f)\right\|_{L^{2}}^{2} & =\int_{\mathbb{R}^{n}} \int_{0}^{\infty}\left|N_{\varepsilon}^{\rho} f(x)\right|^{2} \frac{d \varepsilon}{\varepsilon} d x \\
& =\int_{0}^{\infty} \int_{\mathbb{R}^{n}}|\eta(|\varepsilon x|) \hat{f}(x)|^{2} d x \frac{d \varepsilon}{\varepsilon} \\
& =\int_{\mathbb{R}^{n}}|\hat{f}(x)|^{2} \int_{0}^{\infty}|\eta(\varepsilon x)|^{2} \frac{d \varepsilon}{\varepsilon} d x \\
& =\|\hat{f}(x)\|_{L^{2}}^{2} \cdot \int_{0}^{\infty} \eta(r)^{2} \frac{d r}{r} .
\end{aligned}
$$

因而若要证明 (3.1) 式, 只需证明

$$
\int_{0}^{\infty}(\eta(r))^{2} d r / r \leqslant\left(C / m^{\lambda+1}\right)^{2} .
$$


将上述积分分解为两个部分: $\int_{0}^{\infty}=\int_{0}^{m / 2}+\int_{m / 2}^{\infty}$. 对 $\alpha=1-\rho$ 应用定理 1 , 则后一积分为

$$
\int_{m / 2}^{\infty}\left(\frac{1}{r^{\rho}} \int_{0}^{r} \frac{J_{m+\lambda}(t)}{t^{\lambda-\rho+1}} d t\right)^{2} \frac{d r}{r} \leqslant \frac{C}{m^{2 \lambda-2 \rho+2}} \int_{m / 2}^{\infty} \frac{1}{r^{2 \rho+1}} d r \leqslant \frac{C}{m^{2 \lambda+2}} .
$$

至于前一积分, 我们首先说明 $J_{m+\lambda}(r) / r^{\lambda}$ 在 $0<r<m / 2$ 上关于 $r$ 是递增的. 利用性质 (a) 和 (b) 可得

$$
\left(\frac{J_{\nu}(r)}{r^{\lambda}}\right)^{\prime}=\frac{J_{\nu}^{\prime}(r)}{r^{\lambda}}-\lambda \frac{J_{\nu}(r)}{r^{\lambda+1}}=-\frac{J_{\nu+1}(r)}{r^{\lambda}}+m \frac{J_{\nu}(r)}{r^{\lambda+1}}
$$

方便起见, 记 $\nu=m+\lambda$. 从而只要 $m J_{\nu}(r) / r \geqslant J_{\nu+1}(r), J_{m+\lambda}(r) / r^{\lambda}$ 就是递增的. 对 $\mu=0$ 运用 Bessel 函数的性质 $(\mathrm{e})$, 有

$$
J_{\nu+1}(r)=r \int_{0}^{1} J_{\nu}(r s) s^{\nu+1} d s .
$$

由于 $J_{\nu}(x)$ 在 $0<x<\nu$ 是递增的 ${ }^{[9]}$, 由 (3.4) 式得

$$
J_{\nu+1}(r) \leqslant r J_{\nu}(r) \int_{0}^{1} s^{\nu+1} d s \leqslant r \frac{J_{\nu}(r)}{\nu+2} \quad(0<r<\nu) .
$$

若 $0<r<m / 2$, 显然 $0<r<\nu$ 且 $r /(\nu+2) \leqslant m / r$, 因而

$$
J_{\nu+1}(r) \leqslant r \frac{J_{\nu}(r)}{\nu+2} \leqslant \frac{m J_{\nu}(r)}{r},
$$

且 $\left(J_{\nu}(r) / r^{\nu}\right)^{\prime} \geqslant 0$. 因此由 $(2.5)$ 和 $(2.6)$ 式有

$$
\begin{aligned}
\int_{0}^{m / 2}(\eta(r))^{2} \frac{d r}{r} & \leqslant \int_{0}^{m / 2}\left(\frac{J_{m+\lambda}(r)}{r^{\lambda}}\right)^{2}\left(\frac{1}{r^{\rho}} \int_{0}^{r} \frac{1}{t^{-\rho+1}} d t\right)^{2} \frac{d r}{r} \\
& \leqslant C \int_{0}^{m / 2}\left(\frac{J_{m+\lambda}(r)}{r^{\lambda}}\right)^{2} \frac{d r}{r} \\
& \leqslant C \int_{0}^{m / 2} \frac{r^{2 m-1}}{\left(2^{m+\lambda} \Gamma(m+\lambda+1 / 2)\right)^{2}} d r \\
& \leqslant C \frac{1}{2 m}\left(\frac{\left(\frac{m}{2}\right)^{m}}{2^{m+\lambda} \Gamma(m+\lambda+1 / 2)}\right)^{2} \\
& \leqslant C \frac{m^{2 m-1}}{2^{2 m+2 \lambda+2 m+1}(\Gamma(m+\lambda+1 / 2))^{2}} \\
& \leqslant C \frac{m^{2 m-1}}{2^{4 m+2 \lambda+1} \cdot 2 \pi(m+\lambda+1 / 2)^{2(m+\lambda)} \mathrm{e}^{-2 m-2 \lambda-1}} \\
& \leqslant C \frac{\mathrm{e}^{2 m+2 \lambda+1} \cdot m^{2 m-1} \cdot(m+\lambda+1 / 2)}{4^{2 m+2 \lambda+1} 2^{-2 \lambda} \pi(m+\lambda+1 / 2)^{2 m+2 \lambda+1}} \\
& \leqslant C\left(\frac{\mathrm{e}}{4}\right)^{2 m+2 \lambda+1} \quad(m+\lambda+1 / 2) \frac{1}{(m+\lambda+1 / 2)^{2 \lambda+2}} \cdot \frac{m^{2 m-1}}{(m+\lambda+1 / 2)^{2 m-1}} \\
& \leqslant C \frac{1}{m^{2 \lambda+2}} .
\end{aligned}
$$

由 (3.3) 和 (3.5) 式可以得到 (3.2) 式, 从而证明了引理.

我们将运用下面一个经典结果 (见文献 [10, p. 158] 中定理 3.10) 来完成定理 2 的证明.

定理 $\mathbf{C}$ 若 $n \geqslant 2$ 且 $f \in L^{1}\left(\mathbb{R}^{n}\right) \cap L^{2}\left(\mathbb{R}^{n}\right)$ 具有 $f(x)=f_{0}(|x|) P(x)$ 的形式, 其中 $P(x)$ 是 $m$ 阶球体调和函数, 则 $f$ 的 Fourier 变换为 $\hat{f}(x)=F_{0}(|x|) P(x)$, 这里

$$
F_{0}(r)=2 \pi i^{-m} r^{-(n+2 m-2) / 2} \int_{0}^{\infty} f_{0}(s) J_{(n+2 m-2) / 2}(2 \pi r s) s^{(n+2 m) / 2} d s,
$$


$r=|x|, J_{\nu}$ 是 Bessel 函数.

定理 2 的证明 证明的基本思想取自文献 [11]. 定义 $S^{n-1}$ 上 $m$ 阶球面调和函数空间 为 $\mathcal{H}_{m}$, 其维数为 $\mathcal{D}_{m}$. 通过极限过程, 我们仅需考虑 $f \in C_{0}^{\infty}\left(\mathbb{R}^{n}\right)$ 的情形. 记

$$
\Omega\left(x, z^{\prime}\right)=\sum_{m \geqslant 0} \sum_{j=1}^{\mathcal{D}_{m}} a_{m, j}(x) Y_{m, j}\left(z^{\prime}\right),
$$

其中对于 $m \geqslant 0$ 和 $j=1,2, \ldots, \mathcal{D}_{m},\left\{Y_{m, j}\right\}$ 为完全标准正交球面调和函数系. 注意到 $\Omega\left(x, z^{\prime}\right)$ 满足 (1.3) 式, 因此 $a_{0, j} \equiv 0$. 定义

$$
a_{m}(x)=\left(\sum_{j=1}^{\mathcal{D}_{m}}\left|a_{m, j}(x)\right|^{2}\right)^{1 / 2} \quad, \quad b_{m, j}(x)=\frac{a_{m, j}(x)}{a_{m}(x)},
$$

则

$$
\sum_{j=1}^{\mathcal{D}_{m}} b_{m, j}^{2}(x)=1
$$

且

$$
\Omega\left(x, z^{\prime}\right)=\sum_{m \geqslant 1} a_{m}(x) \sum_{j=1}^{\mathcal{D}_{m}} b_{m, j}(x) Y_{m, j}\left(z^{\prime}\right) .
$$

如果记

$$
\mu_{m, j}^{\rho} f(x)=\left(\int_{0}^{\infty}\left|\int_{|x-y| \leqslant t} \frac{Y_{m, j}(x-y)}{|x-y|^{n-\rho}} f(y) d y\right|^{2} \frac{d t}{t^{2 \rho+1}}\right)^{1 / 2},
$$

则应用 Hölder 不等式两次以及 (3.6) 式, 有

$$
\begin{aligned}
\left(\mu_{\Omega}^{\rho} f(x)\right)^{2}= & \int_{0}^{\infty}\left|\int_{|x-y| \leqslant t} \sum_{m \geqslant 1} a_{m}(x) \sum_{j=1}^{\mathcal{D}_{m}} b_{m, j}(x) \frac{Y_{m, j}(x-y)}{|x-y|^{n-\rho}} f(y) d y\right|^{2} \frac{d t}{t^{2 \rho+1}} \\
\leqslant & \left(\sum_{m \geqslant 1} a_{m}^{2}(x) m^{-\varepsilon}\right)\left(\sum_{m \geqslant 1} m^{\varepsilon} \int_{0}^{\infty}\left|\int_{|x-y| \leqslant t} \sum_{j=1}^{\mathcal{D}_{m}} b_{m, j}(x) \frac{Y_{m, j}(x-y)}{|x-y|^{n-\rho}} f(y) d y\right|^{2} \frac{d t}{t^{2 \rho+1}}\right) \\
\leqslant & \left(\sum_{m \geqslant 1} a_{m}^{2}(x) m^{-\varepsilon}\right) \\
& \cdot\left(\sum_{m \geqslant 1} m^{\varepsilon} \int_{0}^{\infty}\left(\sum_{j=1}^{\mathcal{D}_{m}} b_{m, j}^{2}(x)\right) \cdot \sum_{j=1}^{\mathcal{D}_{m}}\left|\int_{|x-y| \leqslant t} \frac{Y_{m, j}(x-y)}{|x-y|^{n-\rho}} f(y) d y\right|^{2} \frac{d t}{t^{2 \rho+1}}\right) \\
\leqslant & \left(\sum_{m \geqslant 1} a_{m}^{2}(x) m^{-\varepsilon}\right) \cdot\left(\sum_{m \geqslant 1} m^{\varepsilon} \sum_{j=1}^{\mathcal{D}_{m}}\left(\mu_{m, j}^{\rho} f(x)\right)^{2}\right),
\end{aligned}
$$

其中 $0<\varepsilon<1$. 由文献 $[11$, p. 230] 中 (4.4) 式, 当 $\varepsilon$ 充分靠近 1 且 $q>2(n-1) / n$ 时,

$$
\left(\sum_{m \geqslant 1} a_{m}^{2}(x) m^{-\varepsilon}\right)^{1 / 2} \leqslant C\left(\int_{S^{n-1}}\left|\Omega\left(x, z^{\prime}\right)\right|^{q} d \sigma\left(z^{\prime}\right)\right)^{1 / q} \leqslant C\|\Omega\|_{L^{\infty}\left(\mathbb{R}^{n}\right) \times L^{q}\left(S^{n-1}\right)} .
$$

应用 Minkowski 不等式和 (3.7) 式, 有

$$
\left\|\mu_{\Omega}^{\rho} f\right\|_{L^{2}}^{2} \leqslant C\|\Omega\|_{L^{\infty}\left(\mathbb{R}^{n}\right) \times L^{q}\left(S^{n-1}\right)}^{2} \cdot \sum_{m \geqslant 1}\left(m^{\varepsilon} \sum_{j=1}^{\mathcal{D}_{m}}\left\|\mu_{m, j}^{\rho} f\right\|_{L^{2}}^{2}\right),
$$


其中 $0<\varepsilon<1$. 为了完成定理 2 的证明, 只需说明

$$
\sum_{j=1}^{\mathcal{D}_{m}}\left\|\mu_{m, j}^{\rho} f\right\|_{L^{2}}^{2} \leqslant C m^{-2}\|f\|_{L^{2}}^{2} .
$$

令 $\varphi_{t, \rho}^{m, j}(x)=t^{-\rho} Y_{m, j}\left(x^{\prime}\right)|x|^{-n+\rho} \chi_{\{|x| \leqslant t\}}(x)$. 由 Plancherel 定理有

$$
\left\|\mu_{m, j}^{\rho} f\right\|_{L^{2}}^{2}=\int_{0}^{\infty} \int_{\mathbb{R}^{n}}\left|\varphi_{t, \rho}^{m, j}(x) * f(x)\right|^{2} d x \frac{d t}{t}=\int_{0}^{\infty} \int_{\mathbb{R}^{n}}\left|\left(\varphi_{t, \rho}^{m, j} * f\right)^{\wedge}(\xi)\right|^{2} d \xi \frac{d t}{t} .
$$

现在令 $P_{m, j}(x)=Y_{m, j}\left(x^{\prime}\right)|x|^{m}$, 则 $P_{m, j}$ 为 $m$ 阶球体调和函数且

$$
\varphi_{t, \rho}^{m, j}(x)=t^{-\rho} P_{m, j}(x)|x|^{-n-m+\rho} \chi_{\{|x| \leqslant t\}}(x) .
$$

显然

$$
\psi_{0}(|x|)=t^{-\rho}|x|^{-n-m+\rho} \chi_{\{|x| \leqslant t\}}(|x|)
$$

是固定 $t>0$ 时关于 $x$ 的径向函数. 由定理 $\mathrm{C}$

$$
\left(\varphi_{t, \rho}^{m, j}\right)^{\wedge}(\xi)=F_{0}(|\xi|) P_{m, j}(|\xi|)=Y_{m, j}\left(\xi^{\prime}\right)|\xi|^{m} F_{0}(|\xi|),
$$

其中

$$
\begin{aligned}
F_{0}(r) & =2 \pi i^{-m} r^{-[(n+2 m-2) / 2]} \int_{0}^{\infty} \psi_{0}(s) J_{(n+2 m-2) / 2}(2 \pi r s) s^{(n+2 m) / 2} d s \\
& =2 \pi i^{-m} r^{-[(n+2 m-2) / 2]} \int_{0}^{t} t^{-\rho} s^{-n-m+\rho} J_{(n+2 m-2) / 2}(2 \pi r s) s^{(n+2 m) / 2} d s \\
& =2 \pi i^{-m} t^{-\rho} r^{-[(n+2 m-2) / 2]} \int_{0}^{t} s^{-n / 2+\rho} J_{(n+2 m-2) / 2}(2 \pi r s) d s \\
& =2 \pi i^{-m} t^{-\rho} r^{(-m-n / 2)} \int_{0}^{2 \pi r t} \theta^{-n / 2+\rho}(2 \pi r)^{-\rho+n / 2} J_{(n+2 m-2) / 2}(\theta) d \theta \\
& =i^{-m} r^{-m}(2 \pi)^{n / 2} \frac{1}{(2 \pi r t)^{\rho}} \int_{0}^{2 \pi r t} \frac{J_{(n+2 m-2) / 2}(\theta)}{\theta^{n / 2-\rho}} d \theta .
\end{aligned}
$$

由 (3.10) 和 (3.11) 式,

$$
\left(\varphi_{t, \rho}^{m, j} * f\right)^{\wedge}(\xi)=i^{-m}(2 \pi)^{n / 2} Y_{m, j}\left(\xi^{\prime}\right) \frac{1}{(2 \pi|\xi| t)^{\rho}} \int_{0}^{2 \pi|\xi| t} \frac{J_{(n+2 m-2) / 2}(\theta)}{\theta^{n / 2-\rho}} d \theta \cdot \hat{f}(\xi) .
$$

因此

$$
\begin{aligned}
\sum_{j=1}^{\mathcal{D}_{m}}\left\|\mu_{m, j}^{\rho} f\right\|_{L^{2}}^{2} & =\int_{\mathbb{R}^{n}} \int_{0}^{\infty} \sum_{j=1}^{\mathcal{D}_{m}}\left|\left(\varphi_{t, \rho}^{m, j} * f\right)^{\wedge}(\xi)\right|^{2} \frac{d t}{t} d \xi \\
& =(2 \pi)^{n} \int_{\mathbb{R}^{n}} \int_{0}^{\infty} \sum_{j=1}^{\mathcal{D}_{m}}\left|Y_{m, j}\left(\xi^{\prime}\right)\right|^{2} \cdot\left|\frac{1}{(2 \pi|\xi| t)^{\rho}} \int_{0}^{2 \pi|\xi| t} \frac{J_{(n+2 m-2) / 2}(\theta)}{\theta^{n / 2-\rho}} d \theta \cdot \hat{f}(\xi)\right|^{2} \frac{d t}{t} d \xi
\end{aligned}
$$

由于

$$
\sum_{j=1}^{\mathcal{D}_{m}}\left|Y_{m, j}\left(\xi^{\prime}\right)\right|^{2}=\omega^{-1} \mathcal{D}_{m} \sim m^{n-2}
$$

其中 $\omega$ 为 $S^{n-1}$ 的面积 (见文献 [12, p. 225] 中 (2.6) 式). 可得

$$
\sum_{j=1}^{\mathcal{D}_{m}}\left\|\mu_{m, j}^{\rho} f\right\|_{L^{2}}^{2} \leqslant C m^{n-2} \int_{\mathbb{R}^{n}} \int_{0}^{\infty}\left|\frac{1}{(2 \pi|\xi| t)^{\rho}} \int_{0}^{2 \pi|\xi| t} \frac{J_{(n+2 m-2) / 2}(\theta)}{\theta^{n / 2-\rho}} d \theta \cdot \hat{f}(\xi)\right|^{2} \frac{d t}{t} d \xi .
$$

由 (3.12) 式和引理 3.1 可以推出 (3.9) 式. 这样完成了定理的证明. 
注 3.1 应用定理 2 的结论和文献 [7] 中的思想, 可以得到分别与 Lusin 面积积分 $S$ 和 Littlewood-Paley $g_{\lambda}^{*}$ 函数相关的变量核的参数型 Littlewood-Paley 型算子 $\mu_{\Omega, S}^{\rho}$ 和 $\mu_{\Omega, \lambda}^{\rho, *}$ 的 $L^{2}$ 有界性. 这里 $\mu_{\Omega, S}^{\rho}(f)(x)$ 和 $\mu_{\Omega, \lambda}^{*, \rho}(f)(x)$ 分别定义为

$$
\mu_{\Omega, S}^{\rho}(f)(x)=\left(\iint_{\Gamma(x)}\left|F_{\Omega, t}^{\rho}(y)\right|^{2} \frac{d y d t}{t^{n+1}}\right)^{1 / 2}
$$

和

$$
\mu_{\Omega, \lambda}^{*, \rho}(f)(x)=\left(\iint_{\mathbb{R}_{+}^{n+1}}\left(\frac{t}{t+|x+y|}\right)^{n \lambda}\left|F_{\Omega, t}^{\rho}(y)\right|^{2} \frac{d y d t}{t^{n+1}}\right)^{1 / 2}, \quad \lambda>1,
$$

其中

$$
F_{\Omega, t}^{\rho}(x)=\frac{1}{t^{\rho}} \int_{|x-y| \leqslant t} \frac{\Omega(x, x-y)}{|x-y|^{n-\rho}} f(y) d y, \quad 0<\rho<n,
$$

并且 $\Gamma(x)=\left\{(y, t) \in \mathbb{R}_{+}^{n+1}:|x-y| \leqslant t\right\}$.

定理 3 若 $0<\rho \leqslant \frac{n}{2}, \Omega \in L^{\infty}\left(\mathbb{R}^{n}\right) \times L^{q}\left(S^{n-1}\right), q>2(n-1) / n$, 且满足(1.3)式, 则

$$
\left\|\mu_{\Omega, S}^{\rho}(f)\right\|_{2} \leqslant 2^{\lambda n}\left\|\mu_{\Omega, \lambda}^{*, \rho}(f)\right\|_{2} \leqslant C_{\lambda, n}\|f\|_{2} .
$$

致谢作者对审稿人的有益建议深表感谢.

\section{参考文献}

1 Calderón A, Zygmund A. On a problem of Mihlin. Trans Amer Math Soc, 78: 209-224 (1955)

2 Stein E. On the function of Littlewood-Paley, Lusin and Marcinkiewicz. Trans Amer Math Soc, 88: 430-466 (1958)

3 Benedek A, Calderón A, Panzone R. Convolution operators on Banach space valued functions. Proc Natl Acad Sci USA, 48: 356-365 (1962)

4 Ding Y, Fan D, Pan Y. Weighted boundedness for a class of rough Marcinkiewicz integrals. Indiana Univ Math J, 48: 1037-1055 (1999)

5 Ding Y, Fan D, Pan Y. $L^{p}$-boundedness of Marcinkiewicz integrals with Hardy space function kernels. Acta Math Sin (Engl Ser), 16: 593-600 (2000)

6 AL-Salman A, AL-Qassem H, Cheng L C, et al. $L^{p}$ bounds for the function of Marcinkiewicz. Math Res Lett, 9: 697-700 (2002)

7 Ding Y, Lin C, Shao S. On the Marcinkiewicz integral with variable kernels. Indiana Univ Math J, 53(3): 805-821 (2004)

8 Hörmander L. Estimates for translation invariant operators in $L^{p}$ spaces. Acta Math, 104: 93-140 (1960)

9 Watson G. A Treatise on the Theory of Bessel Functions. Cambridge: Cambridge University Press, 1966

10 Stein E, Weiss G. Introduction to Fourier Analysis on Euclidean Spaces. Princeton: Princeton University Press, 1971

11 Calderón A, Zygmund A. On singular integrals with variable kernels. Appl Anal, 7: 221-238 (1978)

12 Torchinsky A, Wang S. A note on the Marcinkiewicz integral. Colloq Math, 60-61: 235-243 (1990) 